\title{
ELECTROMAGNETIC ACTUATOR FOR LASER BEAM POSITIONING - MANUFACTURING PROCESS
}

\author{
Cristinel Ilie ${ }^{1}$, Marius Popa ${ }^{1}$, Nicolae Tanase ${ }^{1}$, Ionel Chirita ${ }^{1}$, Dragos Ovezea ${ }^{1}$ \\ ${ }^{1}$ National Institute for Research and Development in Electrical Engineering ICPE-CA, \\ Splaiul Unirii no. 313, 030138, District 3, Bucharest \\ E-mail: cristinel.ilie@icpe-ca.ro, marius.popa@icpe-ca.ro, nicolae.tanase@icpe-ca.ro, ionel.chirita@icpe-ca.ro, \\ dragos.ovezea@icpe-ca.ro
}

\begin{abstract}
The purpose of this paper is to presents the conception, achievements, measurement and data analysis regarding the connecting and the assembling of the components of a MEMS electromagnetic microactuator, cantilever type. The microactuator consists of two main components: a fixed lower part on which a flat coil has been machined and a movable upper part (made using a cantilever) consisting of an array of permanent magnets. The flat coil was made in two constructive variants, spiral type and grid type, made on a glass fibber board wafer covered with a layer of $35 \mu \mathrm{m}$ of $\mathrm{Cu}$. The array of permanent magnets was made using lithography for template and electroplating for deposition of magnetic materials. All process parameters for the exposure, development, etching of the cooper layer and removal of the unexposed photoresist, were optimized. Dimensional measurements were made for the finished parts, comparing them with designed data, and have tried removing the negative effect of isotropic etching. The conclusions established the optimal parameters for the realization of the components
\end{abstract}

Keywords: Microactuator, UV LASER Photolithography, SU8 3050 Resist, Cantilever, MEMS, Micromachining, LASER Beam.

\section{Introduction}

It is well known the magnitude of the microelectromechanical systems (MEMS), which penetrate more and more sectors of life, contributing to the achievement of new functions for notifying phenomena, to the acquisition of signals and their processing for low power controls and drives, in increasingly important areas: automotive industry (airbags, safety systems), robotics, aerospace and security, medicine (medical optics, administration and monitoring of drug delivery, endoscopy and microsurgery), industry applications (process control and monitoring), biology applications (cell separation, blood analysis, catheters), information technology (scanning), etc.

Besides integrated circuits, MEMS devices create new opportunities in the development of sensors and actuators based on mechanical, electrical, electrostatic and electromagnetic, chemical and biological principles for various applications: optical, microfluidics, accelerometers, gyroscopes, microphones and geophones or combinations thereof.

MEMS systems operating on magnetic principles can generate higher forces than other systems and operate at greater distances than those based on electrostatic principles, as the energy densities provided are much more important.
In addition, the lack of contact between the parts reduces the influence of frictional forces and increases the dynamic performance. Moreover, they are less susceptible to malfunctions when working in unfavourable environments - such as dust and humidity - and can be operated with low-cost voltage controllers [1-6].

The development of electromagnetic microactuators was stimulated by the development of LIGA technology and the SU8 family of photoresist, which allowed the development of thick-layered MEMS applications, of the order of hundreds of micrometres.

This paper presents the manufacturing technology for processing the components of an MEMS electromagnetic microactuator, whose destination is the precise positioning of a laser beam, used in microsurgery.

The microactuator is composed of two main components: a fixed lower part on which a flat coil has been machined and a movable upper part containing an array of permanent magnets. The mobility of the upper part is given by a cantilever.

\section{Technical Solution}

The technical solution chosen for the microactuators is presented in Figure 1. 


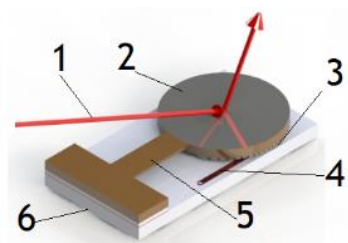

a) Microactuator with flat spiral coil - assembly

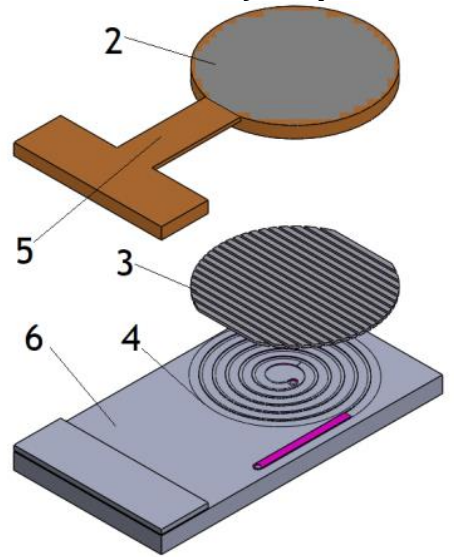

b) Microactuator with flat spiral coil - exploded

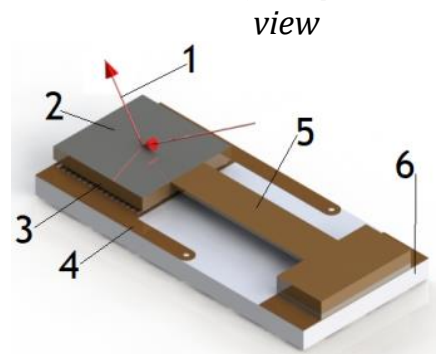

c) Microactuator with grid with current paths assembly
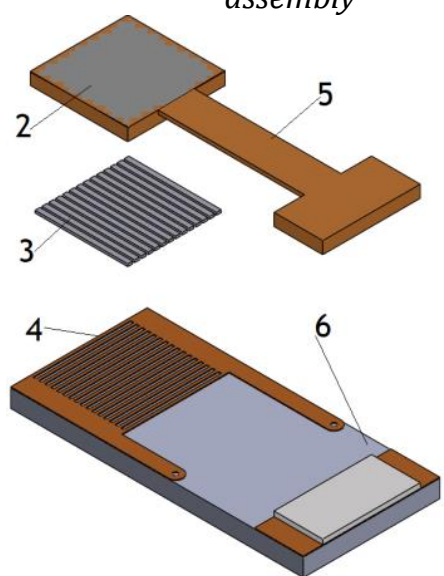

d) Microactuator with grid with current paths exploded view

Figure 1: Electromagnetic micro actuator with flat spiral coil $(a, b)$ and electromagnetic micro actuator with grid with parallel current paths $(c, d) ; 1$-laser beam, 2-mirror surface, 3-micromagnets, 4-coil / grid with parallel current paths ,5-cantilever, 6-support

Two constructive variants were chosen, both operating on the principle of electromagnetic force the interaction between a permanent magnet and a conductor crossed by electric current.
It is observed that in both variants the actuator is composed of an array of permanent magnets 3 , fixed to the cantilever 5 , which interacts with a current path 4 , made on a rigid support 6 . The system of forces that participate to maintain the balance, according to the sketch from Figure 1, is given by the electromagnetic interaction force between the flat spiral coil (or grid with parallel current paths) and the array of the permanent magnets and mechanical elastic force given by the cantilever. The deflection of the mirror 2 is given for different positions of the balance between the elastic force of the cantilever and the electromagnetic force between the electric current path and the array of the permanent magnets.

The variant with the grid with parallel current paths was chosen due to the fact that this solution is closer to the classical theory of electromagnetic force, in this case the direction of the force being perpendicular to the microactuator plane.

\section{The Manufacturing Process of the Flat Spiral Coil / Grid with Parallel Current Paths}

The manufacturing process (Figure 2) started from a rigid glass fibber board wafer (2), covered with a layer of copper (1), which is also covered with a thin layer of photoresist (3). In this photoresist layer was configured the future form of the current path, the flat spiral coil or the grid with parallel current paths.

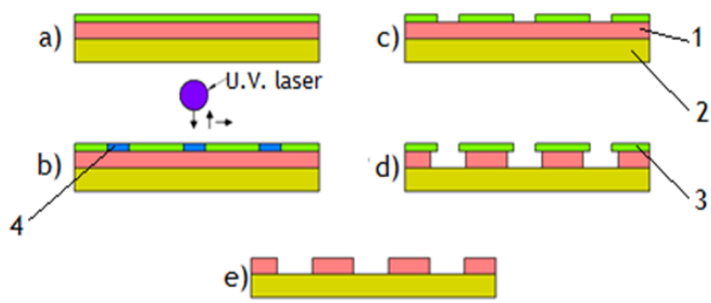

Figure 2: The technological process to obtain the current paths

The main steps of the manufacturing process shown in Figure 2 are [1]:

a) The glass fibber board wafer covered with $\mathrm{Cu}$ (1- $35 \mu \mathrm{m}$ thick copper layer; 2 - glass fibber board wafer; 3- thin layer of photoresist);

b) laser lithography (4-photoresist exposed);

c) development;

d) chemical corrosion of the copper layer;

e) removal of the unexposed photoresist.

For the photoresist was used Positive 20 type spray, which reacts at a wavelength of $310-440 \mathrm{~nm}$ (purple).

For the lithography process, a specialized equipment was used, the direct writing UV laser system, type DWL66FS. This system allowed the transfer of the drawings of the parts we intended to 
manufacture, from the computer on which they were made, to the work wafer.

The direct writing system uses a matrix scanning technology to expose the substrate. The exposure time, for a certain configuration of a drawing, does not depend on the filling factor of the respective structure, as in the vector scanning technology, but only on the entire area of the drawing [7]. Direct laser writing removes the inconveniences of using masks related to the manufacturing time and mask costs, as well as the possibility of an uneven gap between the mask and the photoresist layer [8]. In this way there are no disturbances related to light diffraction. The system we use is equipped with a laser diode with an average power of $18 \mathrm{~mW}$ and a wavelength of $375 \mathrm{~nm}$ (UV). The $4 \mathrm{~mm}$ writing head is a satisfactory compromise between precision and writing speed (accuracy of $1 \mu \mathrm{m}$ at a writing speed of $5.7 \mathrm{~mm}^{2} / \mathrm{min}$ ) [9].

All process parameters were optimized for exposure, development, corrosion of the copper layer and removal of the unexposed photoresist.

Miniature current paths were manufactured, in the shape of a flat spiral coil and of a current grid with parallel paths (Figure 3). They are processed on a $\mathrm{Cu}$-coated glass fibber board wafer.

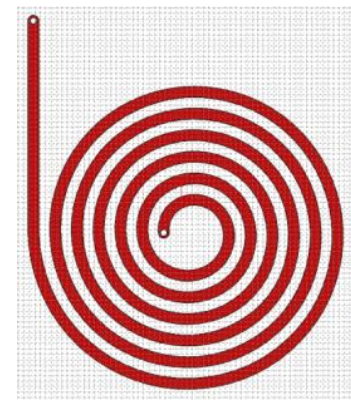

a) Flat spiral coil;

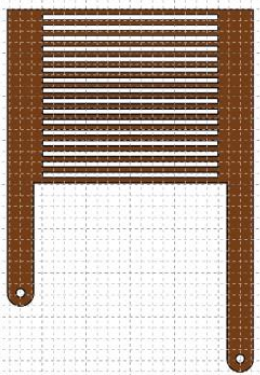

b) Grid with parallel current paths.
Figure 3: Miniature current paths - drawings

A range of microstructures with flat spiral coils was made with the following characteristics: Number of turns: 7; Coil thickness: $0.035 \mathrm{~mm}$; Coil width: $0.22 \mathrm{~mm}$; Distance between turns: $0.22 \mathrm{~mm}$.

Current grid-type microstructures with parallel paths were also made with the following characteristics: Number of current paths: 17; Coil thickness: $0.035 \mathrm{~mm}$; Coil width: $0.12 \mathrm{~mm}$; Distance between turns: $0.12 \mathrm{~mm}$.

The overall dimensions being similar for both microparts, at the same exposure can be obtained 25 pieces, arranged in a $5 \times 5$ matrix.

\subsection{Optimization of the Process Parameters. Experimental Results.}

For all the operations of the technological process it was necessary to test and optimize the process parameters, in real conditions and benefiting from the available equipment. Throughout the experiments, by optimal value was meant that value of a process parameter that were used to obtain the best image of the designed structure. The approach followed a logical technological cycle, each time modifying a single process parameter. After determining the optimal value for the tested parameter, we proceeded to optimize the next parameter and so on. Exposure, development, corrosion of the copper layer and removal of the unexposed photoresist were performed in a special chamber in yellow light to avoid unwanted exposure of the photoresist to light.

The manufacturing process involved the following technological operations, in accordance with the steps of the technological process from b) to e) steps, presented in Figure 2:

Step b) - The laser lithography meant performing the following activities:

b1) making the manufacturing drawing of the current paths (flat spiral coil and current grid with parallel paths), in a format accepted by the laser lithography system DWL66FS, (DXF, CIF, Gerber, GDSII);

b2) the conversion of the manufacturing drawing into a dot matrix (LIC) and the positioning of each element to be fabricated on the support in one of the fields of a matrix (MAP file), which contains in a matrix format a number of fields of equal size, which forms a map in which LIC files are placed, here of $5 \mathrm{x}$ 5 elements;

b3) setting the parameters for exposure (to complete the JOB file), which contains the name and path of each LIC file to be exposed (DESIGN), the energy with which the exposure is made, expressed as a percentage of the maximum energy (ENERGY), the focus expressed as a numerical value between 0 and 4095 (DEFOC) and the exposure command (DO = -1) or configuration only (DO $\neq-1)$;

b4) the exposure using the DWL66FS laser lithography system, which involves loading the system by placing the photoresist wafer to be displayed on the machine table and fixing it to the vacuum system, focusing by FOCUS control and views the clear image of the wafer on one from the two monitoring cameras of the system; automatic identification of the center of the wafer and manual or automatic alignment, depending on the application; the effective exposure and unloading of the system by removing the plate that was exposed from the machine table.

The technological operation from step b3) was optimized, thus the following parameters were optimized: Optical filter. During the exposure we used optical filters with different values: No filter, $50 \%$ filter and $25 \%$ filter. The optimal value was: Filter 50\%. Software filter. We used different values for the software filter (percentage of the 
maximum exposure energy): 60, 70, 80, 90, $100 \%$. The optimal value was: $90 \%$. DEFOC software parameter. During the exposure process, different values were used for the DEFOC software parameter (a fine adjustment of the distance between the writing head and the substrate). Values from 2000 to 4000 were used in steps of 50 units. The optimal value was: 2350.

\section{Step c) - Development:}

c1) for the development of the exposed photoresist, the following parameters have been optimized:

Development time and type: The development of the exposed photoresist was performed by immersion in $\mathrm{NaOH}$ type solvent 7 gr./l. Experiments were performed by modifying the immersion time of the wafers with microstructures in solvent and experiments with the intercalation of the rinsing operation with deionized water to remove the photoresist from the small channels. The development temperature was the ambient temperature $(21-25){ }^{\circ} \mathrm{C}$, with testing starting from a still process, without shaking the developer, and continuing with a vigorous stirring, of course going through intermediate stages. The samples were kept in the developer from $1 \mathrm{~min}$ to $4 \mathrm{~min}$. With and without agitation. Optimal value: 2 min. and $15 \mathrm{~s}$, with gentle stirring;

c2) rinsing and drying: Drying type: natural or by forced convection (air jet). Optimal value: natural. The final rinsing was done with deionized water and drying was done using compressed air or simply waiting, using natural convection.

\section{Step d) - Etching of the copper layer:}

The following parameters were optimized:

\section{d1) Time and type of etching:}

The samples were maintained in the developer from $15 \mathrm{~min}$ to $40 \mathrm{~min}$, with and without shaking them. Optimal values: $26 \mathrm{~min}$., with mild shaking, for flat spiral coil and and $35 \mathrm{~min}$, for grid with parallel current paths.

d2) Drying type: natural or forced convection (air jet). Optimal value: natural.

The etching of the cooper layer was achieved by immersion in $\mathrm{FeCl} 340 \%$, solvent type. They have been made experiments, ranging the immersion time of the microstructure in the solvent, and experiments with the intercalation of rinses in deionized water, to remove the resist from the smallsized channels. The etching temperature was the room temperature $\left(21-25^{\circ} \mathrm{C}\right)$, and there were made etching without shaking, with vigorous stirring and intermediate variants. The final rinse was done with distilled water and the drying was realized with compressed air and natural convection.

\section{Step e) Removal of the unexposed photoresist:}

e1) removal of the unexposed photoresist - was performed by immersion acetone; e2) rinsing and drying. The rinsing was done with deionized water and drying was done using compressed air or simply waiting, using natural convection.

\subsection{Determination of Dimensional Accuracy}

A ZEISS Semi C2000 optical microscope was used to obtain high-precision measurements.

The measurements were made on a set of 25 microparts for each category. The standard deviation was less than $10 \mu \mathrm{m}$. The dimensional accuracy of the microparts is strongly influenced by the accuracy of the technological process parameters. When designing the sensors, the effects of isotropy during the corrosion process of the copper layer were taken into account.

The following examples (Figure 4 and Figure 5) show us the differences between measured and projected values.

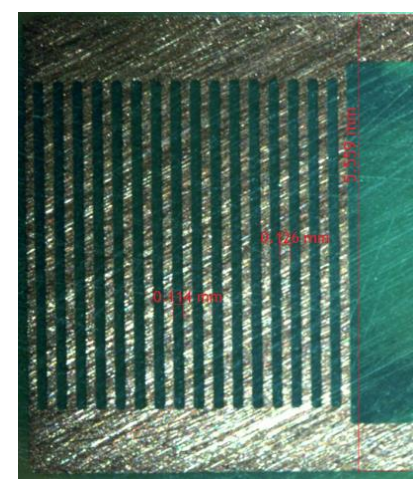

The width of the active area of the current paths; Designed: $5550 \mu \mathrm{m}$ Measured: $5559 \mu \mathrm{m}$. Copper path width: Designed: $120 \mu \mathrm{m}$ Measured: $126 \mu \mathrm{m}$ Distance between current paths: Designed: $120 \mu \mathrm{m}$ Measured: $114 \mu \mathrm{m}$

Figure 4: Grid with parallel current paths; Optical microscopy, X12.5

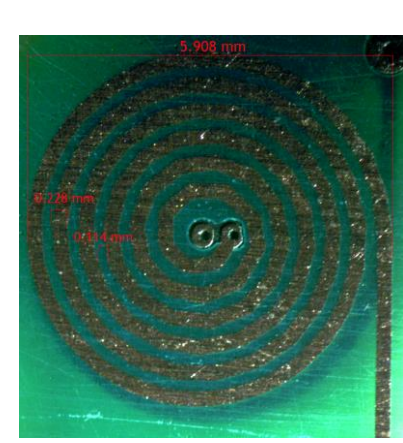

Diameter of the active area of the coil: Designed: $5900 \mu \mathrm{m}$ Measured: $5908 \mu \mathrm{m}$. Copper path width: Designed: $220 \mu \mathrm{m}$ Measured: $128 \mu \mathrm{m}$ Distance between current paths: Designed: $220 \mu \mathrm{m}$ Measured: $114 \mu \mathrm{m}$

Figure 5: Flat spiral coil; Optical microscopy, X12.5

The differences between the designed dimensions, those estimated during the design process and those obtained are presented in Table 1. For example, in order to obtain a width of the copper path with a size of $220 \mu \mathrm{m}$, it was necessary to provide a compensation of the nominal size in the execution drawing, which would be able to compensate the effects of isotropy during the copper corrosion process. 
Thus, the nominal dimension inscribed on the drawing was $290 \mu \mathrm{m}$, which compensates the isotope corrosion on both sides of the copper path, finally obtaining $228 \mu \mathrm{m}$.

Table 1. Differences between the designed dimensions

\begin{tabular}{|c|c|c|c|}
\hline Dimensions & $\begin{array}{c}\text { Manufactured } \\
{[\mu \mathrm{m}]}\end{array}$ & $\begin{array}{c}\text { Estimated } \\
{[\mu \mathrm{m}]}\end{array}$ & $\begin{array}{c}\text { The size on } \\
\text { the } \\
\text { manufacturing } \\
\text { drawing }[\mu \mathrm{m}]\end{array}$ \\
\hline \multicolumn{4}{|c|}{ Grid with parallel current paths } \\
\hline $\begin{array}{c}\text { Copper } \\
\text { path width }\end{array}$ & 126 & 120 & 190 \\
\hline $\begin{array}{c}\text { Copper } \\
\text { paths } \\
\text { spacing }\end{array}$ & 114 & 120 & 50 \\
\hline Grid width & 5559 & 5550 & 5620 \\
\hline \multicolumn{3}{|c|}{ Flat coil } & 290 \\
\hline $\begin{array}{c}\text { Copper } \\
\text { path width }\end{array}$ & 228 & 220 & 150 \\
\hline $\begin{array}{c}\text { Copper } \\
\text { paths } \\
\text { spacing }\end{array}$ & 114 & 220 & 59700 \\
\hline \begin{tabular}{c} 
Grid width \\
\hline
\end{tabular} & 5908 & 5900 & 290 \\
\hline
\end{tabular}

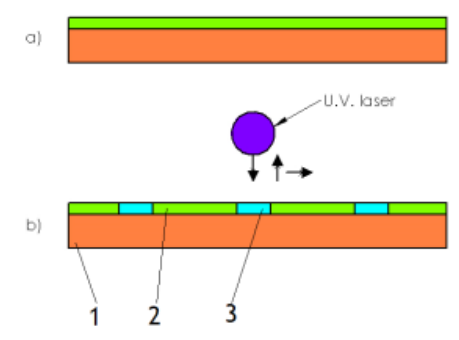

In order to establish the optimal technology for the fabrication of the current paths, it is recommended to optimize the exposure, development and corrosion processes. For this reason, concrete solutions have been proposed that can provide an appropriate accuracy, the differences between the design data and those measured being of the order of micrometers.

\section{Manufacturing of the Permanent Magnets}

The technological process of manufacturing permanent magnets is based on the first two steps of LIGA technology: lithography and electroplating/ electroforming. For lithography, the same laser system with direct writing, DWL type is used, as in the construction of the current paths. Through it, the pattern of the future configurations of the micro magnets is made. Those will be fabricated using an electroforming process. The manufacturing process (Figure 6) started from a rigid brass plate (2), covered with a layer of SU8 photoresist, (3). The future shape of the permanent magnet arrays was configured in this photoresist layer.

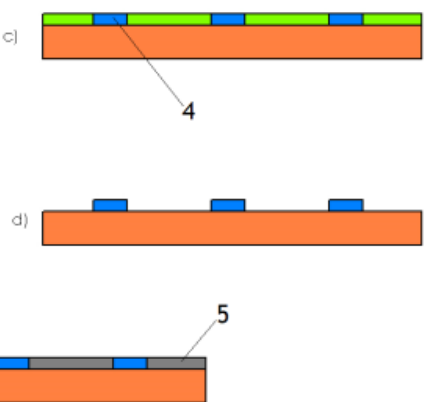

Figure 6: The technological process for micromagnets manufacturing
The main steps of the manufacturing process shown in Figure 6 are [1]:

a) brass plate covered with photoresist (1-brass plate; 2- photoresist);

b) lithography (3-photoresist exposed);

c) post-exposure baking (4-photoresist exposed and thermally stabilized);

d) development;

e) galvanic deposition of permanent magnets.

Compared to the previous technological process, another type of photoresist was used that allows the manufacturing of large thicknesses of parts, of the order of hundreds of $\mu \mathrm{m}$, from the SU8 family. It was used SU8 3050 type photoresist, that has a maximum sensitivity at a wavelength of $365 \mathrm{~nm}$ (UV) [10]. The use of this type of photoresist involves an additional technological operation when lithography is performed, namely post-exposure baking. The postexposure baking aims to stabilize the SU8 structures.

The lamellar and array structures of micromagnets were made on a brass plate covered with SU8, being able to obtain, at the same exposure, 25 microparts, arranged in a matrix of $5 \times 5$, according to Figure 7.

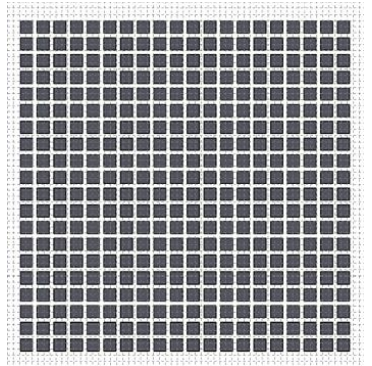

a) Array structure;

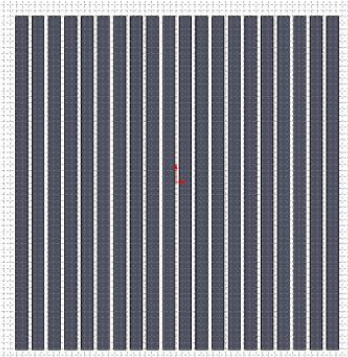

b) Lamellar structure.
Figure 7: Array and lamellar structures - drawings

The magnets array contains structures with the size of $200 \times 200 \mu \mathrm{m}$, the distance between them being $80 \mu \mathrm{m}$. The overall dimensions for the matrix with 20x20 microstructures is $5,520 \mathrm{~mm}$. The lamellar structures have a width of $200 \mu \mathrm{m}$, the 
distance between them being $80 \mu \mathrm{m}$. The overall dimensions are identical to the above.

The manufacturing of the structures for the micromagnet arrays involved the achievement and optimization the lithography and development operations.

\subsection{Optimization of Process Parameters for Lithography}

The optimization of the process parameters for Lithography meant the exposure without optical filter and the value of the software filter, (percentage of maximum energy), equal to 100 . The software parameter for correcting the focus, DEFOC (fine adjustment of the distance between the writing head and the substrate) was 2500 and 2600 .

The temperature and baking time PE1 were 1 min. at $65{ }^{\circ} \mathrm{C}$, made on a thermostatic hob type FALC MOD F 70, and the temperature and baking time PE2 were $5 \mathrm{~min}$. at $95{ }^{\circ} \mathrm{C}$, made in a forced convection oven type Binder FD70, placed on a metal support with high thermal inertia.

\subsection{Optimization of Process Parameters for Development}

The development of the photoresist SU8 was performed by immersion in solvent type mr-Dev600. Experiments were performed varying the maintenance time of the microstructures in the solvent, but also experiments with the intercalation of rinses in 2-propanol (ICAO: isopropanol) to remove the photoresist from the small dimensions channels.

The development temperature was the room temperature $\left(21-25^{\circ} \mathrm{C}\right)$, experiencing developments without agitation of the developer, with strong agitation and intermediate variants. They have made several attempts. Thus, the developments were performed immediately after the sample reached room temperature or after a period of relaxation of the structures. The final rinsing was performed with 2-propanol and drying was done with compressed air jet or natural convection.

Optimal results involved a development time of 7 min. with gentle stirring, followed by rinsing in isopropyl alcohol. Then 2 min. with gentle stirring, followed by another rinse with isopropyl alcohol. Finally, a rinse with deionized water was done, followed by a natural drying, at room temperature.

\subsection{Practical Results}

Conclusive images with structures during the Exposure and Development optimization process are presented in Figure 8. The arrays contain structures with the size of $200 \times 200 \mu \mathrm{m}$, the distance between them being $80 \mu \mathrm{m}$.

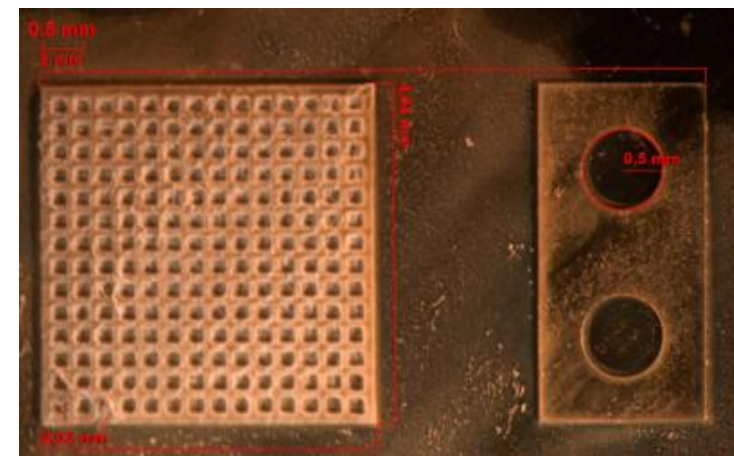

a) $4 \times 4 \mathrm{~mm}$ matrix, optical microscopy $x 10$;

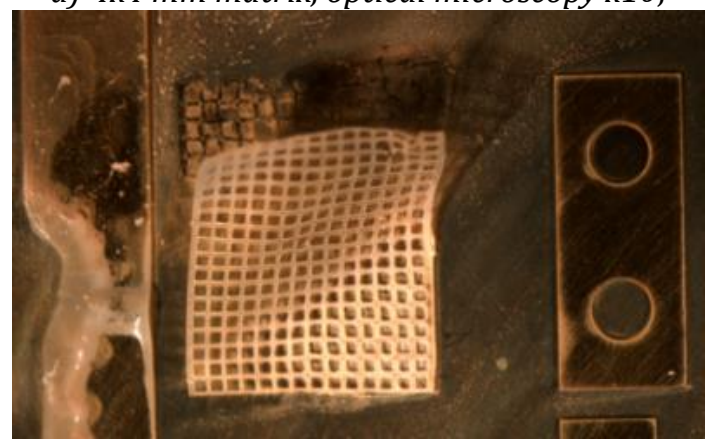

b) Exfoliated matrix, optical microscopy x10;
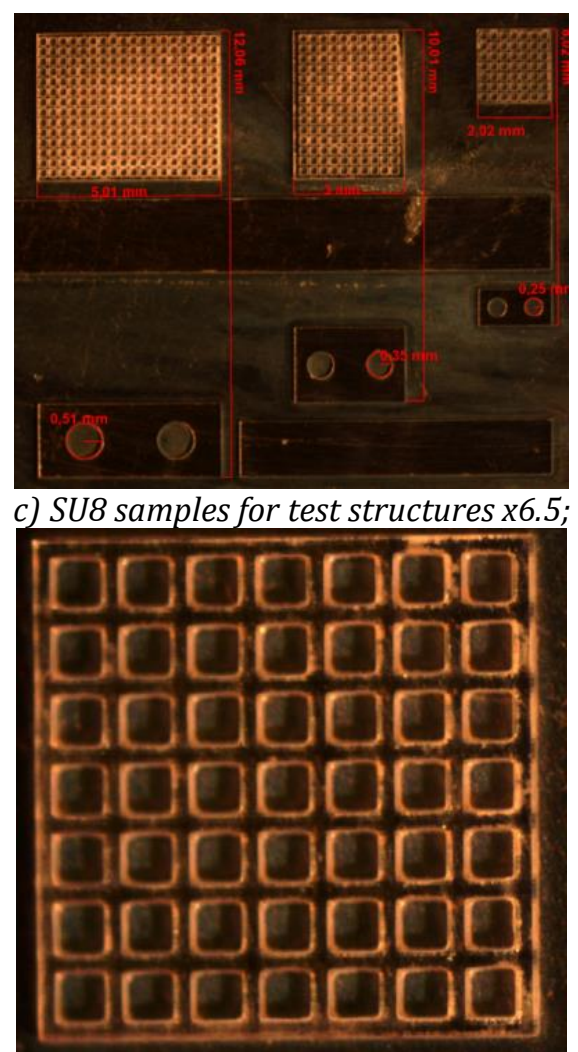

d) $2 \times 2 \mathrm{~mm}$ matrix, optical microscopy $x 40$.

Figure 8: Samples for magnet arrays structures

Images with structures during the Exposure and Development optimization process are presented in Figure 9. The matrices contain lamellar structures with a width of $200 \mu \mathrm{m}$, the distance between them being $80 \mu \mathrm{m}$. 


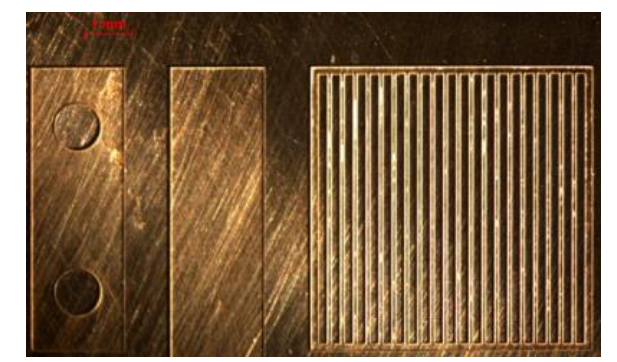

a) $6 \times 6 \mathrm{~mm}$ array, optical microscopy $x 10$;

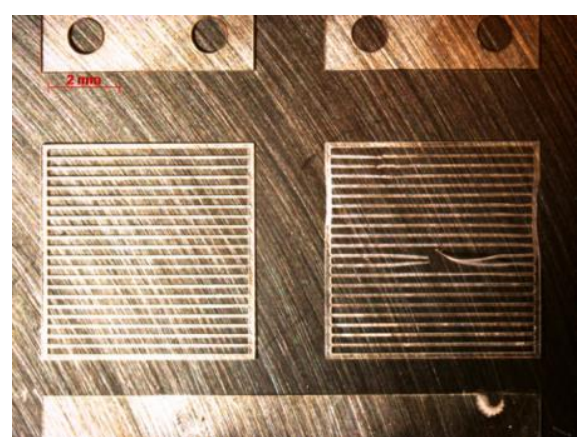

c) Detail with damaged sample: rupture and exfoliation, light microscopy $x 8$;

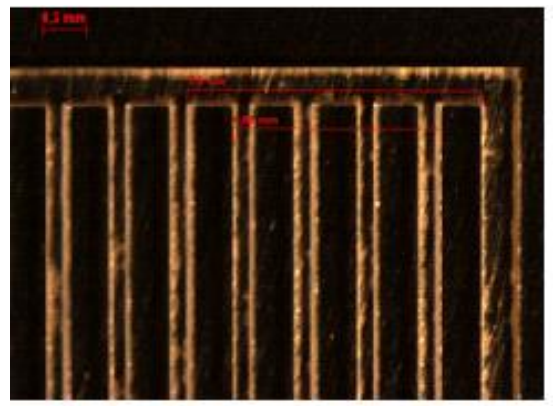

b) Optical microscopy detail x50;

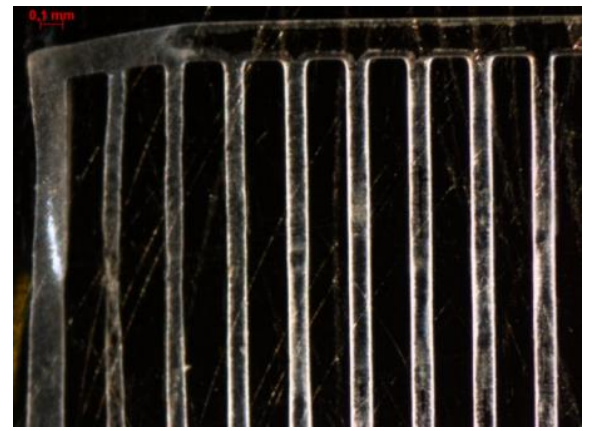

d) Detail with exfoliation $\times 50$;

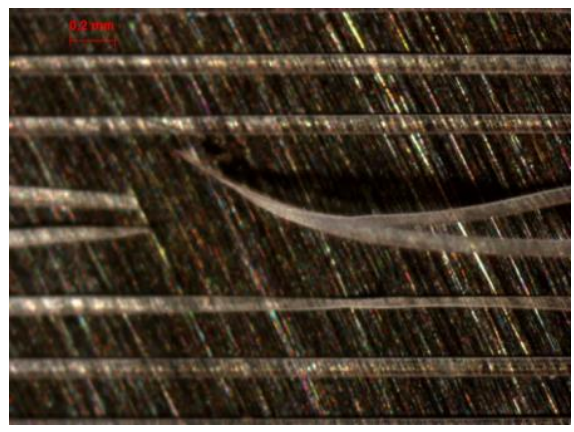

e) Detail with rupture $\times 50$.

Figure 9: Samples for lamellar array structures

It can be seen that the structures of the arrays presented in Figure 10 are qualitatively superior to the previous ones.

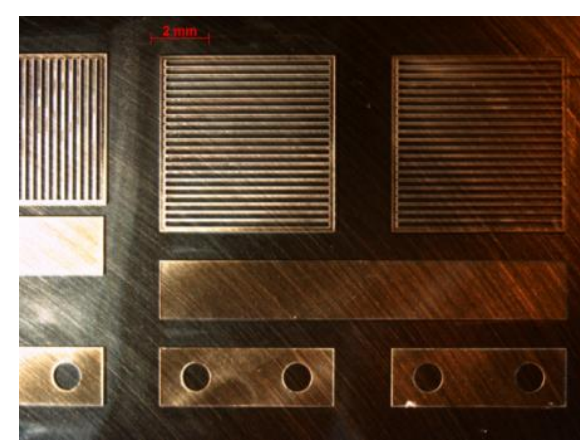

Figure 10: Samples for lamellar array structures, optical microscopy $x 6,5$

\subsection{Electrodeposition}

Electrodeposition metals and alloys is a useful and inexpensive technique to produce magnetic structures on a substrate of a predefined shape.
Thin films of permanent magnets containing rare earth metals are commonly used in practice, although they have a high coercivity and remanence and require additional heat treatment at about 600 ${ }^{\circ} \mathrm{C}$ to align the magnetic moment by recrystallization, which is inconvenient for the postprocessing process of electronic devices. A disadvantage is that these films cannot be made in defined forms [11]. In addition, rare earth magnets oxidize and degrade easily.

This shortcoming has led, as an alternative, to the electrodeposition of the magnetic alloys with cobalt nickel films [11-14]. The deposition can be obtained by electrolysis (electrochemical deposition) or chemical deposition (electroless). In the chemical deposition (electroless) more uniform and much more resistant to corrosion films are made. However, this process has very slow deposition rates and the electrolyte is unstable over time. Regarding the integration of magnetic materials in microelectromechanical systems (MEMS), it is preferable to obtain the films by electrodeposition 
(electrochemical deposition). The electrodeposition technique is viable due to the low cost, high flow rate and high quality of the deposit obtained [15-16].

In addition, the properties of the materials can be "adapted" by controlling the solution compositions and deposition parameters [17-18]. The magnetic thin films have good adhesion, are resistant to corrosion and are thermally stable with excellent magnetic properties. One way to increase the stored energy is to increase the volume of the magnet, i.e. the thickness of the film. This (thick layers) is possible if UV LIGA technology based on SU8 photoresist is used [19].

\subsection{Experiments for Electrodeposition of CoNiPMn Type Magnetic Alloy}

Galvanostatic deposition, in which a constant current is applied without potential control, is the most commonly used technique for NiCo-based alloy electrodeposition processes. The advantage of this method is that it does not require complex equipment, it does not need a potentiostat or reference electrodes. It works only with a deposition bath, a standard cathode and a direct current source [20].

The operating parameters in the electrodeposition of NiCoMnP alloys to be controlled are divided into two categories: (i) the parameters involved in metal deposition, as parameters of the electrolyte bath (electrolyte, precursor concentration, buffer, additives, pH) and temperature; (ii) parameters affecting the uniformity, structure and texture of the deposit: current density and application of different current pulses, geometry and configuration of the substrate, stirring of the solution, magnetic field during electroplating. In order to establish and verify the deposition conditions and the physico-chemical and magnetic characteristics of CoNiMnP alloys type, preliminary deposition experiments were performed on different substrates: brass of different sizes with or without magnetic field and $\mathrm{Cu}$ foil. Before starting the process of electrodeposition of $\mathrm{Cu}$ and brass metal substrates, their surface was prepared by the following sequences:

- degreasing in organic solvents (acetone, ethyl alcohol), $\mathrm{T}=25-30{ }^{0} \mathrm{C}$, immersion time about $120 \mathrm{sec}$.

- chemical pickling / deoxidation in HNO3 1:1 (vol.), $\mathrm{T}=25-30{ }^{\circ} \mathrm{C}$; immersion time $30-60 \mathrm{~s}$.

Between the two sequences the sample was rinsed and dried. The electrodeposition process was performed using a stabilized current source of $1 \mathrm{~A}$ and $30 \mathrm{~V}$.

The thickness of the electrodeposited NiCo layer $(\delta)$ was determined gravimetrically. The electrolyte used to deposit the NiCoMnP alloy in the magnetic field has the composition shown in Table 2.
Table 2. The composition of the electrolyte used for deposition

\begin{tabular}{|l|l|}
\hline Compound & Gram / liter \\
\hline $\mathrm{CoCl}_{2} \cdot 6 \mathrm{H}_{2} \mathrm{O}$ & 26 \\
\hline $\mathrm{NiCl}_{2} .6 \mathrm{H}_{2} \mathrm{O}$ & 24 \\
\hline $\mathrm{MnSO}_{4} \cdot \mathrm{H}_{2} \mathrm{O}$ & 3.6 \\
\hline $\mathrm{NaH}_{2} \mathrm{PO}_{2} \cdot \mathrm{H}_{2} \mathrm{O}$ & 4.6 \\
\hline $\mathrm{B}(\mathrm{OH})_{3}$ & 24 \\
\hline $\mathrm{NaCl}$ & 23 \\
\hline Saccharin & 0.8 \\
\hline Sodium lauryl sulfate & 0.1 \\
\hline Cee $\left(\mathrm{SO}_{4}\right)_{3}$ & 0.01 \\
\hline
\end{tabular}

A bath with two electrodes was used, which contains the copper and brass substrates as the cathode and both $\mathrm{Ni}$ and Co plates, with a large surface area as soluble anodes. The surface of the copper and brass electrodes (anodes) before the electrodeposition of the film was gradually polished with carbon paper, degreased with acetone and then washed with deionized water and dried.

A mechanical stirring of the electrolyte was performed to avoid diffusive control and mass transfer to the cathode and to work with high current densities.

The electrolysis vessel was surrounded with permanent magnets to obtain magnetic films with oriented structures, with the best possible performance, (Figure 11) [21].

After electrolysis, the cathode was removed from the vessel and the NiCoMnP deposit was washed well with deionized water and dried.

The cathodic current efficiency was calculated from the amount of NiCoMnP alloy deposited at the cathode after electrolysis, taking into account Faraday's law and using the number of electrons transferred $\mathrm{z}=2$.

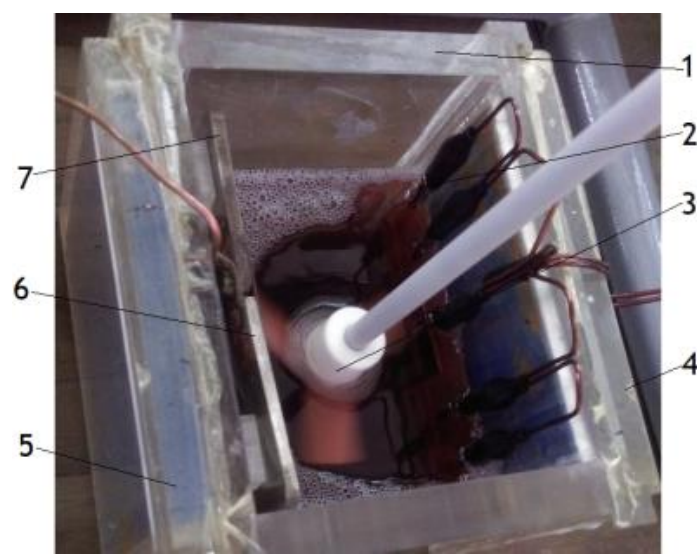

Figure 11: Two electrodes electrolytic bath;

1-tank; 2-cathodes (coating samples);

3-mechanical stirrer; 4, 5-permanent magnets; 6- $\mathrm{Ni}$ anode; 7- $\mathrm{Co}$ anode

The thickness of the layer was determined knowing the densities of pure $\mathrm{Ni}$ and $\mathrm{Co}, \rho \mathrm{Ni}=8.908$ 
$\mathrm{g} / \mathrm{cm}^{3}, \rho \mathrm{Co}=8.72 \mathrm{~g} / \mathrm{cm}^{3}$ and the measurement of the NiCoMnP deposit surface. Sodium hypophosphite (diacid phosphate) and boric acid are buffers. It is known that in the absence of buffers, at normal temperatures, the metal deposits become brittle and porous. Boric acid helps to form whiter deposits, having at the same time a levelling effect on the deposited layer. Sodium chloride ensures a good electrical conductivity of the solution. Saccharin is a gloss agent (ensures a glossy deposit, but without the character of mirror gloss). Due to the incorporation in the deposited layer of the released hydrogen and of the colloidal metallic hydroxides adsorbed on the cathode, on the deposited surface pores are formed, which constitute the so-called "pitting effect". To avoid this, surfactants are added, the most commonly used being sodium lauryl sulfate.

It reduces the surface tension of the deposition bath, increases wetting and prevents the fixation of hydrogen bubbles or other chemical species on the cathode. The $\mathrm{pH}$ of the deposition solution must be maintained at a value <4.2; above this value, sodium lauryl sulfate loses its effectiveness, and in the bath large amounts of colloidal metal hydroxides are formed, which adsorb the surfactants.

A stabilized current source of $1 \mathrm{~A}$ and $30 \mathrm{~V}$ was used for the electrodeposition process. Deposits were made at room temperature $\left(25^{\circ} \mathrm{C}\right)$ and current density of $2 \mathrm{~mA} / \mathrm{cm}^{2}$, with or without electrolyte agitation.

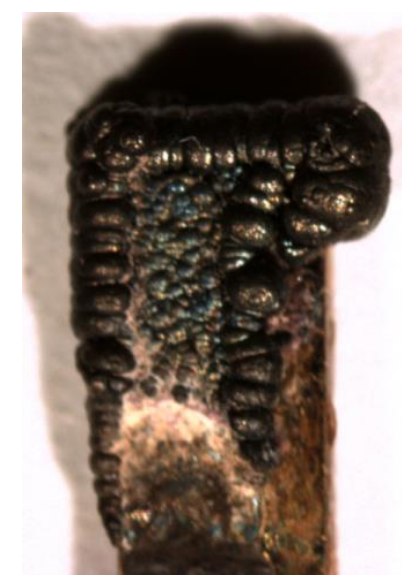

a) Excess deposition, with dendrites, $x 10$ Figure 12: Structures with dendrites and exfoliated structures

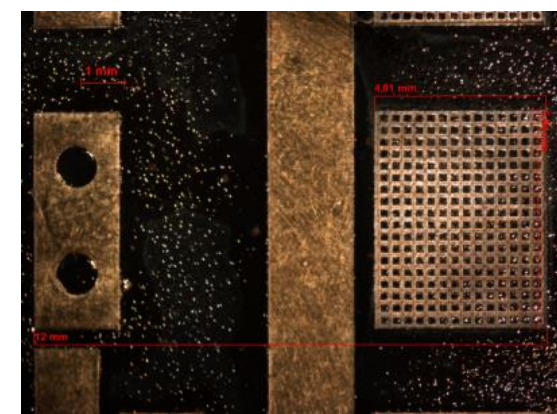

a) $5 \times 4 \mathrm{~mm}$ array, optical microscopy $x 12.5$;
The values of $90-92 \%$ of the current efficiencies were determined gravimetrically. Brass samples with an area of $3 \times 12 \mathrm{~mm}^{2}$ were made, divided into sets of samples. In order to respect the imposed current density and not to decrease the minimum current imposed on the source, in addition to the test structures, an additional sample was added, so that the sample current discharged from the source is at least $22 \mathrm{~mA}$. For all samples, throughout the deposition the electrolyte in the tank was stirred with a mechanical stirrer type Heidolph 2041 with blades, at a speed of $44 \mathrm{rpm}$, the $\mathrm{pH}$ of the electrolyte deposition bath of $25{ }^{\circ} \mathrm{C}$.

The lateral and posterior surface of each sample was protected with varnish so that the magnetic material was deposited only on one side, on an area of approximately $24 \mathrm{~mm}^{2}$. To estimate the thickness of the deposited layer, the samples were weighed before and after deposition. After establishing the optimal parameters, based on the measurements performed on the samples, we proceeded to the galvanic deposition into the photoresist support, thus resulting in the final the proposed microstructures.

\subsection{Practical Results}

Figure 12 and Figure 13 shows comparative images of galvanic deposition on samples.

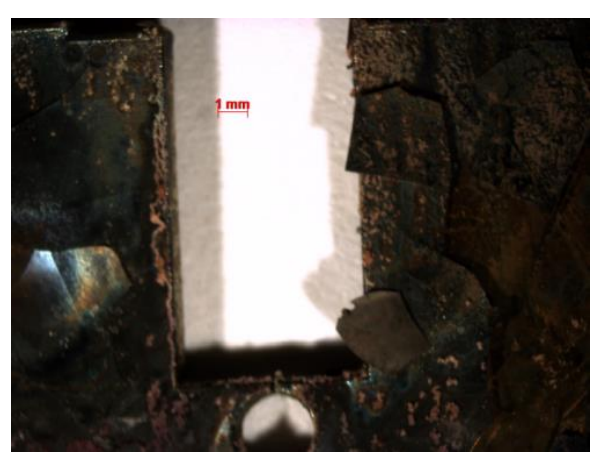

b) Uneven deposits, with exfoliations $x 6.5$ was approx. 2.8 and the temperature of the

Figure 12: Structures with dendrites and exfoliated structures

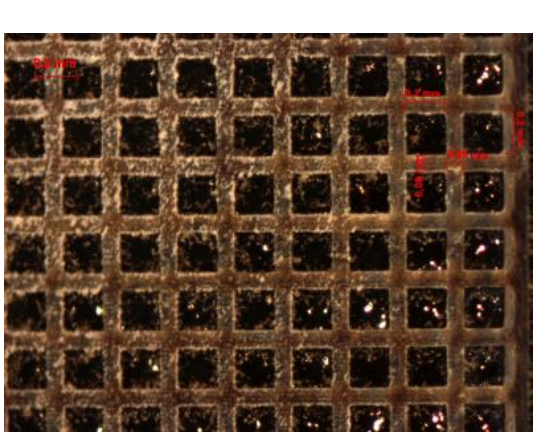

b) Magnets $200 \times 200 \mu m, x 12.5$; 


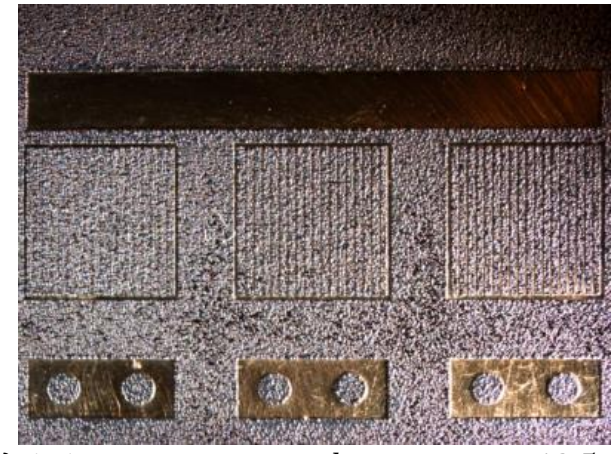

c) $6 \times 6 \mathrm{~mm}$ array, optical microscopy $x 12.5$;

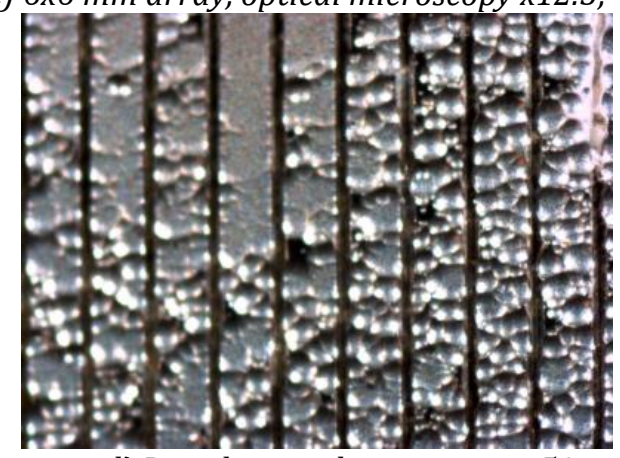

d) Detail, optical microscopy $\times 50$.

Figure 13: Square micromagnets and lamellar micromagnets in arrays arrangements

\section{Conclusions}

Regarding the manufacturing technology, a clear distinction must be made between the one presented in Figure 2 which refers to the manufacturing of the current paths and the one presented in Figure 6 which implies the fabrication of the permanent magnet mats.

The first technology (Figure 2) is generically known as Bulk Micromachining, and has the creation of microdevices by chemical corrosion. Here the photoresist layer is thin, of the order of a few micrometers and the final dimensions of the parts are greatly influenced by the corrosion isotropy. Therefore, the designer must take into account the difference between the dimensions entered in the execution drawings and the estimated final size. This technique is a relatively simple and does not involve large expenses, suitable for applications that do not require much complexity.

The second technology (Figure 6) is based on the first two steps of LIGA technology, lithography and galvanic deposition. Here the deposited photoresist layer has a great importance, in it being "dug" the configuration of the future part, thus making a mold (a sample).

The advantages of this technology consist in the fact that parts thicknesses of several hundred micrometers are possible, with a high aspect ratio, the walls of the dug channels are straight, with smooth surfaces, the precision fabrication being 1 $\mu \mathrm{m}$.
Neither of the two technologies is dependent on the silicon (as in integrated circuit technology).

The electrolytic baths containing cobalt chloride and nickel chloride, which have high penetrating power, buffer capacity and stability, were used for the electrodeposition of nanocrystalline and smooth NiCoMnP films.

In terms of appearance, the NiCoMnP deposits were uniform, fine-grained and glossy. The structures obtained correspond to the proposed purpose.

From the analysis of the manufactured samples it was found that the deposition in the magnetic field is qualitatively superior to the one without field. It is recommended to use the brass plate support.

\section{Acknowledgements}

The authors acknowledge UEFISCDI for the support offered through the 20 PTE/2020 research grant Vibration compensation system of laser beam equipment for microsurgery.

\section{References}

[1] J.W. Judy, R.S. Muller, H.H. Zappe, IEEE J. Microelectromech.Syst. 4 (1995) 162.

[2] Hosaka H., Kuwano H, Yanagia K, "Electromagnetic Microrelays: Concepts and Fundamental Characteristics," Sensors and Actuators A 40: 41-47 (1994).

[3] J.W. Judy, R.S. Muller, Sensors Actuators (Physical A) A 53 (1996) 392.

[4] J.W. Judy, R.S. Muller, IEEE J. Microelectromech. Syst. 6 (1997) 249.

[5] C.H. Ahn, M.G. Allen, IEEE Trans. Ind. Electron. 45 (1998) 866.

[6] H. Guckel, "Progress in Magnetic Microactuators", Mycrosystem Technologies, 5, pp. 59-61, 1998.

[7] ***, DWL 66FS Reference Manual, 2007, Heidelberg Instruments.

[8] Schulz, J., Study concerning metal microstructure processing using LIGA technology, 2008.

[9] ***, DWL 66FS Product Description, 2007, Heidelberg Instruments.

[10]***, NANO SU8 - Negative Tone Photoresist, Technical specifications, 2002, Micro Chem.

[11] D. Golodnitsky, Y. Rosenberg, A. Ulus, Role of anion additives in the electrodeposition of $\mathrm{Ni}$ Co alloy from sulfamate electrolyte, Electrochim. Acta, 47, 2002, pp. 2707-2714

[12] D. Kim, D.Y. Park, B. Y. Yoo, P. T. A. Sumodjo, N. V. Myung, Magnetic properties of nanocrystalline iron group thin film alloys electrodeposited from sulfate and chloride baths, Electrochim. Acta, 48, 2003, pp. 819-830.

[13] C.K. Chung, W.T. Chang, Effect of pulse frequency and current density on anomalous composition and nanomechanical property of 
electrodeposited Ni-Co films, Thin Solid Films, 617 (17), 2009, pp. 4800-4804.

[14] L. Qin, J. Lian, Q. Jiang, Enhanced ductility of high-strength electrodeposited nanocrystalline $\mathrm{Ni}-\mathrm{Co}$ alloy with fine grain size. J. Alloys Compd., 504 (1), 2010, pp. 439-442.

[15] P. Sudhakar, B.S.S. Daniel, P. Jeevanandam, Synthesis of nanocrystalline Co-Ni alloys by precursor approach and studies on their magnetic properties, J. Magn. Magn. Mater., 323 (17) 2011, pp. 2271-2280.

[16] J. V. Arenas, T. Treeratanaphitak, M. Pritzker, Electrochim. Formation of Co-Ni alloy coatings under direct current, pulse current and pulsereverse plating conditions, Acta, 62 (15), 2012, pp. 63-72.

[17] T.M. Liakopoulos, M. Xu, C.H. Ahn, Technical Digest Solid-State Sensor and Actuator Workshop, Vol. 19, Hilton Head Island, SC, USA, 1998.
[18] T.M.Liakopoulos, C.H.Ahn, Proc. Electrochem. Soc.: The 5th Int.Symp. Magnetic Materials, Processes and Devices, Boston, MA, 1998, pp. 413

[19] J.D.Williams, Design and fabrication of electromagnetic micro-relays using the UV LIGA technique, M.Sci. thesis, Louisiana State University, 1996.

[20] Paula PRIOTEASA, Cristinel ILIE, Marius POPA, Mihai IORDOC, Beatrice-Gabriela SBARCEA, Electrodeposition of Nickel for Fabrication of Microfluidic Pumps, REVISTA de CHIMIE (Bucharest) $\downarrow$ Vol. $64 \diamond$ No. $3 \diamond$ 2013.p. 275280,

[21] H.J.Cho, S.Bhansali, C.H.Ahn, Electroplated thick permanent magnet arrays with controlled direction of magnetization for MEMS application, J. Appl. Phys., vol. 87 (9), 2000, pp. 6340-6342. 